\title{
Pseudo tumor cerebri caused by Brucellosis: Case report
}

\author{
Mitra Ranjbar ${ }^{1}$, Sohrab Aghabeigi ${ }^{2 *}$, Fatemeh Tahanian ${ }^{3}$, Ahmad Hezarjaribi $^{2}$ and Sara Nemati ${ }^{4}$ \\ ${ }^{1}$ Department of Infectious Diseases, Iran University of Medical Sciences, Tehran, Iran \\ ${ }^{2}$ Department of Internal Medicine, Iran University of Medical Sciences, Tehran, Iran \\ ${ }^{3}$ Student Research Committee, Faculty of Medicine, Iran University of Medical Sciences, Tehran, Iran \\ ${ }^{4}$ Department of Gynocology and Obstetrics, Shahid Beheshti University of Medical Sciences, Tehran, Iran
}

\begin{abstract}
Brucellosis is a bacterial zoonotic infection. In endemic areas, brucellosis can manifest with various types of neurological symptoms. Among them, meningitis is the most frequent presentation. Pseudotumor like presentation is also rarely reported. In this report, the patient is a 47-year-old female presenting with pseudotumor cerebri and normal cerebrospinal fluid analysis who finally diagnosed with brucellosis. This case report suggests that neurobrucellosis as a differential diagnosis of psudotumor cerebri especially in endemic areas.
\end{abstract}

\section{Introduction}

Brucellosis is one of the most common zoonotic infections in the world [1]; and also is endemic in Iran with 10/100000 annual incidence rate [2]. The disease is transmitted to humans by use of unpasteurized milk and dairy or infected meat [3]. Human brucellosis is traditionally described as a disease of variable manifestations. However, fever is invariable and can be spiking and accompanied by rigors, if bacteremia is present, or may be relapsing mild, or protracted. Malodorous perspiration is almost pathognomonic. Constitutional symptoms are generally present. Physical examination is generally nonspecific, though lymphadenopathy, hepatomegaly, or splenomegaly is often present [4]. Brucellosis has a wide spectrum of clinical manifestation and involves multiple systems and organs. one of the uncommon ones is nervous system involvement, called Neurobrucellosis which only occurs in $5-10 \%$ of patients $[5,6]$. Neurobrucellosis is defined by focal involvement of both central and peripheral nervous system [7]. Central nervous system (CNS) infection is an uncommon but threatening complication of brucellosis which has a different clinical manifestation [8]. Neurological complications are because of both direct and indirect effect of microorganism on the CNS and PNS [9]. meningoencephalitis, meningomyelitis, pseudotumor like presentation, meningovascular presentation, CNS demyelination, peripheral neuritis, compressive myelopathy and radiculopathy secondary to brucella spondylitis; are most common CNS involvement categories [10].

Pseudotumor cerebri (PTC) is characterized by papilledema with increased intracranial pressure, normal cerebrospinal fluid (CSF) composition, and normal neuroimaging findings in the absence of space-occupying lesions or neurologic localizing signs [11].

Brucellosis presenting as pseudotumor cerebri is very rare [12]. Here, we report the a case of neurobrucellosis from Iran who presented with pseudotumor cerebri.

\section{Case report}

The patient was a 47-year-old female admitted to firoozgar hospital for evalluation of five month ataxia. She also had persistent fever without any specific pattern. The patient had been treated with penicillin and acetaminophen previously.
After about a week back pain was added to her symptoms and serological tests were performed for diagnosis of brucellosis and there were all in normal ranges.

Few days later, the fever resolved but the patient started having delirium and became disoriented to time and place. In addition, laboratory findings for collagen vascular diseases such as lupus and RA were all negative. (Table 1).

After a week, the patient started having dizziness too. about two months after the last primary symptoms, the Wright brucellosis test became positive with 1/640 titer (Table 1).

Lumbar puncture (LP) was done for the patient to evaluate brucella infection that were all negative (Table 1).

With the possibility diagnosis of brucella encephalitis, treatment with Streptomycin, Doxycycline, and Rifampin initiated for the patient.

About 20 days later, the patient started blurred vision in her both eyes and development of vertigo. She was unable to walk due to vertigo and impaired balance, Brain magnetic resonance imaging (MRI), Electroencephalography (EEG), audiometery and color doppler ultrasonography of the carotid arteries were done and they were all normal (Figure 1).

With continuation of the dizziness and bilateral papilledema, LP was done again (Table 1) and with the diagnosis of Pseudotumor cerebri, the ventriculo preitoneal shunt was inserted for her. To avoid drug reactions, Streptomycin and Rifampin were discontinued and Cotrimoxazole, Ciprofloxacin, Prednisolone, Doxycycline were prescribed. She was discharged with good general conditions. After 30 days she has been admitted once again with severe respiratory

*Correspondence to: Sohrab Aghabeigi, Department of Internal Medicine, Iran University of Medical Sciences, Tehran, Iran, E-mail: doctorsohrabaghabeigi@gmail.com

Key words: brucellosis, psudotumor cerebri, ICP

Received: November 27, 2019; Accepted: December 11, 2019; Published: December 13, 2019 
Table 1. Laboratory findings

\begin{tabular}{|c|c|c|c|}
\hline \multirow{16}{*}{$\begin{array}{l}\text { Results of serologic } \\
\text { and microbiologic } \\
\text { testing }\end{array}$} & & First admission & After 2 months \\
\hline & WRIGHT & $1 / 640$ & $1 / 640$ \\
\hline & 2ME WRIGHT & $1 / 160$ & $1 / 80$ \\
\hline & $\begin{array}{l}\text { ANTI BRUCELLA } \\
\text { IgG }\end{array}$ & $1 / 5$ & $1 / 5(<9$ neg $)$ \\
\hline & FTA/ABS & neg & neg \\
\hline & $\begin{array}{c}\text { ANTI } \\
\text { CARDIOLIPIN IgG }\end{array}$ & 6.5 & - \\
\hline & $\begin{array}{c}\text { ANTI } \\
\text { PHOSPHOLIPID } \\
\text { Ab IgM }\end{array}$ & neg & - \\
\hline & C-ANCA & neg & - \\
\hline & P-ANCA & neg & - \\
\hline & $\mathrm{C} 4$ & 0.29 & - \\
\hline & $\mathrm{C} 3$ & 1.1 & - \\
\hline & ANTI dsDNA & 7 & - \\
\hline & HIV Ab & neg & neg \\
\hline & $\mathrm{HCV} \mathrm{Ab}$ & neg & neg \\
\hline & HBS Ag & neg & neg \\
\hline & CRP & + & WEAKLY + \\
\hline \multirow{11}{*}{$\begin{array}{l}\text { Results of } \\
\text { cerebrospinal } \\
\text { fluid analysis }\end{array}$} & & First admission & After 20 days \\
\hline & WBC & 40 & 130 \\
\hline & $\mathrm{RBC}$ & 45 & 10 \\
\hline & PROTEIN & 50 & 74 \\
\hline & SUGAR & 77 & 45 \\
\hline & ADA & 37 & 46 \\
\hline & WRIGHT & neg & $1 / 40$ \\
\hline & COOMB'S WRIGHT & neg & neg \\
\hline & VDRL & WEAKLY + & \\
\hline & CULTURE & neg & neg \\
\hline & CSF PRESSURE & - & $300 \mathrm{~mm} \mathrm{H} 2 \mathrm{O}$ \\
\hline
\end{tabular}

distress and due to decreasing SPO2, she was intubated and admitted in intensive care unit (ICU). Because of existence of bicytopenia, the bone marrow aspiration (BMA) was done and it was normocellular and bicytopenia resulting from Cotrimoxazole drug reaction was suggested.

Once again she has recovered from her problems and she was extubated and discharged with good health.

Treatment of brucellosis with Doxycycline and Ciprofloxacin was continued for three months.

In two months and six months follow up after discontinuation of treatment, the patient's general condition was good and she did not have any especial problem.

\section{Discussion}

Neurobrucellosis can cause a variaty of neurologic conditions, such as meningoencephalitic syndrome, vertigo, psychological manifestations, spastic paraplegia, optic neuritis, and radiculitis $[4,13]$. Meningitic presentation is one of the most common neurologic manifestation [10]. Presntation with Pseudotumor cerebri is far less common. for the first time Diaz Espejo et al in 1987 noted the association between pseudotumor cerebri and brucellosis, but their patient had Brucella meningitis with abnormal CSF cytology and destruction of the sella turcica, which was not characteristic of the classic description of PTC [4]. After that a few more case reports of PTC in brucellosis patients were published [7,14-16].

In this case we were suspicious of brucellosis but the first wright brucellosis test results came back negative we repeated the test in about one week and the result was positive with $1 / 640$ titer. This may be due to
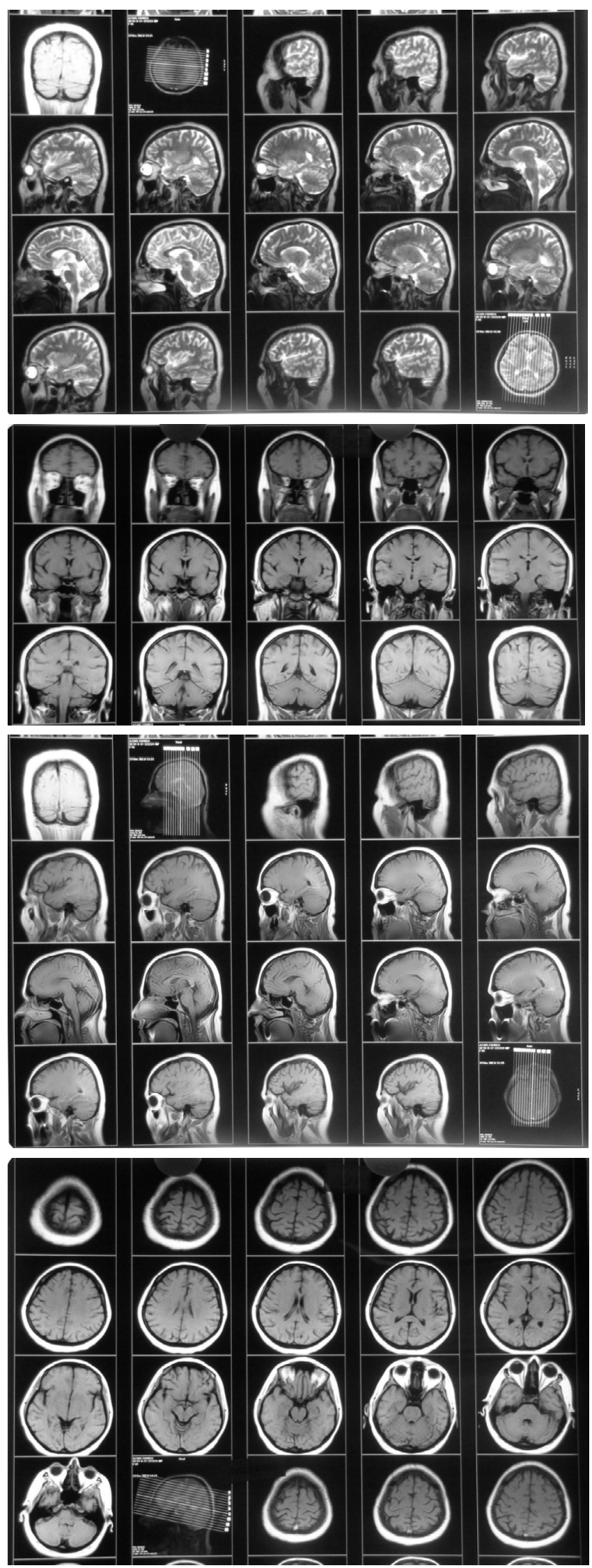

Figure 1. Brain magnetic resonance imaging (MRI) 
the prozone phenomenon, where the high level of antibodies prevents agglutination of the antigen by acting as blocking antibodies resulting in a false-negative test [17].

Most common symptoms in pseudotumor cerebri are headache (92\%), transient visual obscurations $(72 \%)$ and intracranial noises (60\%) [18]. In this case the patient had bllured vision in both eyes and vertigo.

Some features of our case were unique, including normal CSF contents, because studies Showed that CSF is abnormal in almost all patients with neurobrucellosis with lymphocytic pleocytosis of varying degree [19]. The patient also had normal MRI findings.

We reached the diagnosis of brucellosis because the patient had bilateral papilledema not explained by other neurologic disorders, normal CSF contents, positive serologic findings and an adequate response to antibrucellar treatment.

\section{Conclusion}

Neurobrucellosis should be considered in any patient in an endemic area presenting with prolonged illness and associated neurological symptoms. The prozone phenomenon should be kept in mind when the clinical suspicion of brucellosis is strong. Cotrimoxazole drug reactions should be considered when priscribed.

\section{References}

1. Pappas G, Papadimitriou P, Akritidis N, Christou L, Tsianos EV (2006) The new global map of human brucellosis. The Lancet Infectious Diseases 6: 91-99.

2. Mirnejad R, Jazi FM, Mostafaei S, Sedighi M (2017) Epidemiology of brucellosis in Iran: A comprehensive systematic review and meta-analysis study. Microbial Pathogenesis 109: 239-247.

3. Mangalgi SS, Sajjan AG, Mohite ST, Gajul S (2016) Brucellosis in occupationally exposed groups. Journal of Clinical and Diagnostic Research 10: DC24-DC27.

4. Espejo CD, Chaves FV, Ramis BS (1987) Chronic intracranial hypertension secondary to neurobrucellosis. Journal of Neurolog 234: 59-61.
5. Zheng N, Wang W, Zhang J, Cao Y, Shao L, et al. (2017) Neurobrucellosis. International Journal of Neuroscience 128: 55-62.

6. Yetkin MA, Bulut C, Erdinc FS, Oral B, Tulek N, et al. (2006) Evaluation of the clinical presentations in neurobrucellosis. Int J Infect Dis 10: 446-452.

7. Tajdini M, Akbarloo S, Hosseini SM, Parvizi B, Baghani S, et al. (2014) From a simple chronic headache to neurobrucellosis: a case report. Medical Journal of the Islamic Republic of Iran 28: 12.

8. Kanik-Yuksek S, Gulhan B, Ozkaya-Parlakay A, Tezer H (2014) A case of childhood Brucellosis with neurological involvement and epididymo-orchitis. The Journal of Infection in Developing Countries 8: 1636-1638.

9. Asadipooya K, Dehghanian A, Omrani GHR, Abbasi F (2011) Short-course treatment in neurobrucellosis: A study in Iran. Neurology India 59: 101.

10. Kochar DK, Kumawat BL, Agarwal N, Shubharakaran, Aseri S, et al. (2000) Meningoencephalitis in brucellosis. Neurology India 48: 170-173.

11. Johnston I, Hawke S, Halmagyi M, Teo C (1991) The pseudotumor syndrome: disorders of cerebrospinal fluid circulation causing intracranial hypertension without ventriculomegaly. Archives of Neurology 48: 740-747.

12. Ceran N, Turkoglu R, Erdem I, Inan A, Engin D, et al. (2011) Neurobrucellosis: clinical, diagnostic, therapeutic features and outcome. Unusual clinical presentations in an endemic region. Braz J Infect Dis 15: 52-59.

13. Espejo CD, Chaves FV, Ramis BS (1987) Chronic intracranial hypertension secondary to neurobrucellosis. Journal of Neurology 234: 59-61.

14. Güngör K, Bekir NA, Namiduru M (2002) Pseudotumor cerebri complicating brucellosis. Annals of Ophthalmology 34: 67-69.

15. Panagariya A, Sharma B, Mathew V (2007) Pseudotumor--like presentation of neurobrucellosis. The Journal of the Association of Physicians of India 55: 301-302.

16. Sharma PP, Murali MV, Hamdi T (2017) Neurobrucellosis presenting as pseudotumor cerebri: First report from Oman. Oman Medical Journal 32: 507-509.

17. Karsen H, Sokmen N, Duygu F, Binici I, Taskiran H (2011) The false sero-negativity of brucella standard agglutination test: Prozone phenomenon. Journal of Microbiology and Infectious Diseases 1: 110-113.

18. Wall M, George D (1991) Idiopathic intracranial hypertension. A prospective study of 50 patients. Brain 114: 155-180.

19. Saleh M, Al Deeb SM, Yaqub BA (1999) Neurobrucellosis in neurology in tropics. Eds Chopra JS, Sawhney IMS. BI Churchill Livingstone, New Delhi, pp: 345-357.

Copyright: (C2019 Ranjbar M. This is an open-access article distributed under the terms of the Creative Commons Attribution License, which permits unrestricted use, distribution, and reproduction in any medium, provided the original author and source are credited. 